\title{
BMJ Open Efficacy of biological drugs in short- duration versus long-duration inflammatory bowel disease: a protocol for a systematic review and an individual-patient level meta-analysis of randomised controlled trials
}

\author{
Shomron Ben-Horin, ${ }^{1,2}$ Yue Zhao, ${ }^{1}$ Jing Guo, ${ }^{1}$ Ren Mao, ${ }^{1}$ Lena Novack, ${ }^{3}$ \\ Ruslan Sergienko, ${ }^{3}$ Jian Zhang, ${ }^{2}$ Taku Kobayashi, ${ }^{4}$ Toshifumi Hibi, ${ }^{4}$ \\ Yehuda Chowers, ${ }^{5}$ Jean Frederic Colombel, ${ }^{6}$ Laurent Peyrin-Biroulet, ${ }^{7}$ \\ Gilaad Kaplan, ${ }^{8}$ Min-hu Chen ${ }^{1}$
}

To cite: Ben-Horin S, Zhao Y, Guo J, et al. Efficacy of biological drugs in shortduration versus long-duration inflammatory bowel disease: a protocol for a systematic review and an individual-patient level meta-analysis of randomised controlled trials. BMJ Open 2019;9:e024222. doi:10.1136/ bmjopen-2018-024222

- Prepublication history and additional material for this paper are available online. To view these files, please visit the journal online (http://dx.doi. org/10.1136/bmjopen-2018024222).

YZ and JG contributed equally.

Received 21 May 2018

Revised 15 September 2018 Accepted 4 December 2018

Check for updates

(C) Author(s) (or their employer(s)) 2019. Re-use permitted under CC BY-NC. No commercial re-use. See rights and permissions. Published by BMJ.

For numbered affiliations see end of article.

Correspondence to

Shomron Ben-Horin;

shomron.benhorin@gmail.com

\section{ABSTRACT}

Introduction Crohn's disease (CD) and ulcerative colitis (UC) are remitting-relapsing inflammatory diseases often culminating in disease complications and/or need for surgery. Biologic monoclonal antibody drugs ('Biologics') are efficacious for both diseases, but there are no systematic assessments of their efficacy if administered early after disease onset ('top-down' strategy) vis-à-vis later in the course of disease ('step-up' approach).

Methods and analysis Electronic databases (MEDLINE, EMBASE/EMBASE classic Cochrane CENTRAL register of controlled trials, the Cochrane IBD Group Specialised Trials Register and Clinicaltrials.gov registry) will be searched to identify all randomised placebo-controlled clinical trials of food and drug administration (FDA)-approved biologics for CD and UC (by March 2016). Two independent reviewers will screen identified papers, extract data and assess the risk of bias according to the Cochrane Handbook for Systematic Reviews of Interventions. Individual-patientlevel data (IPD) will be extracted from the identified trials through data-sharing platforms for pharmaceutical companies' sponsored trials and by contacting principal investigators of independent investigator-initiated trials. We will analyse induction of remission in patients with early-disease ( $<18$ months since disease onset) versus patients with longer disease duration, using a generalised linear mixed effect model and by a two-stage approach using coefficient for the treatment-by-subgroup interaction within each trial. We will perform receiver operator curve analysis of optimal disease duration for response. Analyses will be separate for CD and UC. This first-of-itskind meta-analysis at IPD level of interaction of disease duration with the response to biologics in UC and CD may elucidate the impact of early initiation of biologics, which is of paramount importance for clinical practice and management strategies of inflammatory bowel disease. Ethics and dissemination This meta-analysis was approved by the Ethics Committee of the First Affiliated Hospital of Sun Yat-sen University. Findings will be
Strengths and limitations of this study

The meta-analysis, whose protocol is described, will be the first to evaluate by individual-patient level data the association between duration of inflammatory bowel disease (IBD) and response to biological drugs, thereby accounting for multiple confounders that may impact the outcome of biologics treatment.

- The meta-analysis will only analyse data from randomised placebo-controlled clinical trials thereby ensuring high-quality source data with standardised outcome measures.

- The meta-analysis will employ data obtained from drug companies for all six FDA-approved biologics for IBD by 2017. This will increase generalisability of the results to multiple therapeutic agents in IBD.

- Limitations of this meta-analysis include the choice to refrain from analysis of data from real-life cohorts, and the non-inclusion of ustekinumab, which was approved for use in Crohn's disease after this study had commenced.

published in peer-reviewed journal and disseminated via scientific meetings and links with organisations.

PROSPERO registration number CRD42018041961.

\section{BACKGROUND}

Crohn's disease (CD) and ulcerative colitis (UC) are chronic immune-driven inflammatory diseases of the gut, collectively known as inflammatory bowel disease (IBD). Understanding of the progressive structural damage to the gut caused by incessant and/or recurrent bouts of inflammation in $\mathrm{CD}$ has led to the hypothesis that early initiation of biological therapy (top-down strategy) may better 
control underlying inflammation and prevent disease progression, compared with a later initiation of these drugs (step-up approach). ${ }^{12}$ This contention has been supported by the SUTD trial which showed clinical benefit for top-down versus step-up treatment with infliximab in patients with $\mathrm{CD} .^{3}$ REACT, a non-blinded controlled cluster randomised trial, did not show clinical benefit but did find lower rate of disease complications among CD patients treated by top-down compared with step-up approach. ${ }^{4}$ However, no trial has directly compared efficacy of biologics in patients with early versus late disease. Such comparison is only available through some post hoc subanalyses of clinical trials and uncontrolled observations in retrospective cohorts. Some,$^{5-7}$ although not all, ${ }^{8}$ of these studies seemed to indicate a better response rate to anti-tumour necrosis factor (TNF) agents among CD patients with early as opposed to late disease. Nonetheless, the impact of duration of $\mathrm{CD}$ on the response to biological therapy has hitherto not been systematically investigated. Furthermore, whether such correlation exists in patients with UC has not been specifically explored.

Therefore, the primary objectives of this study are to investigate the impact of disease duration on the rate of remission induction in $\mathrm{CD}$ and in $\mathrm{UC}$, separately analysed. To this end, we will compare the efficacy of FDA-approved biologics' in patients with early short-term disease versus those with a long-duration of disease.

\section{METHODS AND DESIGN}

This will be a systematic review and meta-analysis performed according to the Cochrane Handbook for Systematic Reviews of Intervention ${ }^{9}$ and the Preferred Reporting Items for Systematic Reviews and Meta-Analyses statement. ${ }^{10}$ The present protocol was devised in accordance with the Preferred Reporting Items for Systematic Reviews and Meta-Analyses Protocol 2015. ${ }^{11}$

\section{PRIMARY OUTCOME}

The primary outcome in the study is induction of remission defined as remission at the end of induction as per the study-specific predefined time point and within 4-14 weeks following initiation of treatment by biologics approved by the FDA for IBD at the time of launching of this study (November 2015). A Crohn's Disease Activity Index $(\mathrm{CDAI})<150$ is defined as remission for assessment of primary outcome of $\mathrm{CD}$ trials, whereas a total Mayo score $\leq 2$ with no individual subscore $>1$ is the primary remission outcome for UC trials. If these scores were unavailable, the remission/response measures are based on the specific clinical score and outcome definition employed by the respective clinical trial.

Induction of remission is preferred over maintenance of remission as the primary outcome of this meta-analysis, reasoning that response to induction more closely reflects underlying IBD biology and its modulation by duration of disease, compared with assessment of continued response during maintenance (which is more prone to confounders unrelated to disease biology per se, such as immunogenicity, compliance and so forth). However, response and remission as well as sustained response will also be analysed as secondary outcomes (see below).

This study will address the following question pertaining to patients, intervention, comparator, outcome (PICO): in adults of more than 18 years of age with $\mathrm{CD}$ or $\mathrm{UC}$ $(\mathrm{P})$, do those who receive biological drugs or placebo when having short-term duration of disease less than or equal to 18 months (I) compared with patients who have long duration ( $>18$ months) of disease $(\mathrm{C})$, have higher proportion of induction of remission by the biological drug compared with placebo $(\mathrm{O})$ ? We hypothesised that the patients with short-term duration of disease will benefit more from the biological drug, that is, the proportion of remission will be higher in these patients compared with patients with long duration of disease.

Of note, this hypothesis as well as the rest of the outcomes will be analysed separately for $\mathrm{CD}$ and UC.

We will analyse the following secondary endpoints in a comparative analysis of patients with short versus long duration of disease.

- The proportion of induction of response: clinical response is defined as CDAI reduction of 100 points from baseline for $\mathrm{CD}$ and as a total Mayo Drop $\geq 30 \%$ AND $\geq 3$ points with either bleeding score of 0 or 1 OR drop of bleeding score $\geq 1$, for UC trials. When these are not available, the response is defined as per the clinical trial's designated response definition.

- The proportion of response and remission at the end of the maintenance phase of the trial (when applicable), at specific time period between weeks 16 and 54 designated for assessment of the maintenance treatment by the trial. These two maintenance outcomes are exploratory given the possible confounding effects of time points of assessments, the heterogeneity across studies in the feed-in cohorts entering maintenance studies and factors such as immunogenicity and compliance which may further confound maintenance treatment success.

- In UC patients: the proportion of colectomy for patients with short versus long duration of disease at the end of the trial.

- Rate of intestinal surgeries and rate of hospitalisations for patients with short versus long duration of disease at the end of the trial.

As a part of a subgroup analysis of the primary outcome, the impact of the class of the biological drug will be assessed by performing a separate subgroup analysis of the primary outcome in patients treated with anti-TNF class of drugs and in patients treated by anti-integrins.

\section{Disease duration definitions}

The definition of short-disease duration ('early disease') is somewhat arbitrary given the absence of evidence or of biological markers that are able to delineate the 
underlying biology of early disease and distinguish it from late disease. In the SONIC trial, for instance, a cut-off of 3 years was adopted in the stratified randomisation to delineate short/long disease duration. ${ }^{12}$ Other published studies and expert opinions' definitions of 'early disease' range between 1 and 5 years since onset. ${ }^{5613-17}$

A recent International Organisation for the Study of Inflammatory Bowel Diseases (IOIBD) working group pragmatically defined early $\mathrm{CD}$ as one that is $\leq 18$ months in duration. ${ }^{18}$ Thus, the present meta-analysis will employ the IOIBD disease duration time point of 18 months to define early versus late disease for the purpose of the primary analysis. However, bearing in mind the chronic life-long course of IBD, the diverse definitions existing, the possibility that patients enrolled into clinical trials at such early stage of their disease are a unique patient group and the paucity of patients with $<18$ months of disease in clinical trials hampering the statistical power of the analysis, we will also perform two sensitivity analyses of the primary outcome and of maintenance of remission, defining short-term disease duration by $\leq 3$ years and by $\leq 5$ years cut-off time points, as well as an receiver operator characteristics (ROC) analysis to define the most accurate point to distinguish disease duration with favourable versus unfavourable response to therapy. For individuals for whom only year-based duration of disease (year of onset) is available rather than month-based time frame, the 1 July of the respective year of disease onset-as the midpoint of the calendric year-was arbitrarily chosen for the imputation of disease duration.

\section{Search strategy and study selection}

Studies were identified by a search of the non-language restricted medical literature conducted with MEDLINE (1976 to November 2015) and EMBASE/EMBASE classic (1946 to November 2015). The full search strategy and search terms are available as online supplementary material. This was further supplemented by a search of the Cochrane CENTRAL register of controlled trials, the Cochrane IBD Group Specialised Trials Register and clinical trials registry (Clinicaltrials.gov) using all developmental and generic drug names, as specified in online supplementary material. Abstract proceedings from Digestive Diseases Week, United European Gastroenterology Week and the European Crohn's and Colitis Organisation between 2007 and November 2015 were hand-searched to identify potentially eligible studies published only in abstract form. Additionally, existing Cochrane systematic reviews were reviewed for relevant referenced trials, ${ }^{19-22}$ AGA technical review ${ }^{23}$ and ECCO guidelines. ${ }^{24}{ }^{25}$ The citation lists of identified relevant studies were used for performing a further cross-search of the literature.

\section{Data extraction and quality assessment}

Titles and/or abstracts of studies retrieved using the search strategy and those from additional sources were screened independently by two review authors (JG and
YZ) to identify studies that potentially meet the inclusion criteria outlined above. The full text of these potentially eligible studies was retrieved and independently assessed for eligibility by two review team members (GZ and SB-H). Any disagreement between two reviewers over the eligibility of particular studies was resolved by joint discussion by four investigators (SB-H, JG, YZ and RM).

Trials' characteristics were derived from the respective publications and CRFs according to predefined data fields to denote: type of study (induction, maintenance and induction+maintenance), number of centres, geographical region, disease type (UC or CD), number of enrolled patients, median disease duration (or mean if median was unavailable), clinical scores used for outcome measurements, time points for induction and/or maintenance outcome determination, percentage with prior anti-TNFa exposure and percentage of patients receiving concomitant immunomodulator. De-identified individual patient level data were received from sponsors of industry-funded RCTs, including the individual treatment allocation, the duration of disease and the various outcome parameters (see below). Data will be received from the companies after data-sharing committees' approval. For investigator-initiated trials which were not industry sponsored or owned, the first and senior authors were contacted to provide the aforementioned information. Tabulation and analysis of data from designated SAS or $\mathrm{R}$ company-specific computerised research environments will be performed. Data will be extracted as per intentionto-treat outcomes unless precluded by trial reporting.

\section{Assessment of risk of bias}

Two investigators independently assessed the risk of bias as described in the Cochrane Handbook for Systematic Reviews of Interventions. ${ }^{26}$ Factors assessed included the following. 1. Sequence generation (ie, was the allocation sequence adequately generated?). 2. Allocation sequence concealment (ie, was allocation adequately concealed?). 3. Blinding (ie, was knowledge of the allocated intervention adequately prevented during the study?). 4 . Incomplete outcome data (ie, were incomplete outcome data adequately addressed?). 5. Selective outcome reporting (ie, are reports of the study free of suggestion of selective outcome reporting?).

6. Other potential sources of bias (ie, was the study apparently free of other problems that could put it at a high risk of bias?). Specifically, because of the nature of disease duration subgroup analysis, potential bias by selective recruitment or allocation of patients with certain disease duration parameters was sought.

Risk of bias was summarised as low risk, high risk or unclear risk of bias per the individual study. Disagreements were discussed and resolved by consensus. Studies with high risk of bias were excluded from the main analysis.

The GRADE criteria were used to assess the overall quality of evidence by: (1) risk of bias from the studies, (2) indirect evidence, (3) inconsistency (unexplained 
heterogeneity), (4) imprecision in data and (5) publication bias. These were assessed as detailed elsewhere by the GRADE consensus. ${ }^{27}$ The overall quality of evidence for each outcome was determined and classified as high quality (ie, further research is very unlikely to change the confidence in the estimate of effect); moderate quality (ie, further research is likely to have an important impact on the confidence in the estimate of effect and may change the estimate); low quality (ie, further research is very likely to have an important impact on the confidence in the estimate of effect and is likely to change the estimate) or very low quality (ie, the estimate is very uncertain). Missing data, consistency of internal data and follow-up duration and outcome was examined, tabulated and compared with the trial protocol and latest trial report or publication.

\section{Data synthesis and statistical analysis \\ General approach in meta-analysis}

In the current analysis, we will employ individual participant data (IPD) meta-analysis, considered the 'gold-standard', provided that the raw data from the trials are available. $^{28} 29$ Two main techniques will be used in the analysis of IPD, known as one-stage and two-stage approaches. Briefly, the one-stage approach employs the methodology of mixed models applied to the full set of IPD, and two-stage approach is based on synthesising the estimates of an effect calculated within each study separately. The one-stage approach is believed to be preferable for estimating patient-level interactions, ${ }^{30} 31$ essential for testing hypotheses in the current project, as it allows for the direct estimation of interactions. The choice of two-stage versus one-stage modelling for the primary analysis depends on the level of heterogeneity of the duration of disease (short term vs long term) - the main covariate in the study-measured by $Q$ estimate as suggested by Simmonds and Higgins, ${ }^{32}$ whereas the two-stage approach is preferred over one-stage approach if $\mathrm{Q} / \mathrm{t}<1$, where $\mathrm{t}$ is the number of studies and $Q$ is a measure of heterogeneity of covariates in each study calculated as follows:

$$
Q=\sum \frac{m_{i}^{2}}{\sigma_{z i}^{2}} \text {, where } \mathrm{m} \text { is the difference of the mean }
$$

covariate value in each study from the overall covariate value and $\sigma$ is the standard deviation of the covariate $(\mathrm{z})$ in each study. For the purpose of completeness of analysis, both two-stage and one-stage approaches will be performed.

We will use variance of the random treatment effect as a measure of heterogeneity, along with $Q$ statistic and $\mathrm{I}^{2}$ estimates, being more intuitive for interpretation, even though the later measures originate from the two-stage meta-analysis approach. To assess whether the variation across trials was due to true heterogeneity or chance, heterogeneity among studies will be evaluated using the Chi-square test (a p-value $\leq 0.10$ considered statistically significant) and the $\mathrm{I}^{2}$ statistic. $\mathrm{I}^{2}$ values range from $0 \%$ to $100 \%$, with $0 \%$ representing no observed heterogeneity. ${ }^{33}$ An $\mathrm{I}^{2}$ value $>50 \%$ is considered substantial.
All analyses in the study will be performed on all available cases from the trials. The missing pattern of the main covariates will be inspected and missing values of outcomes and main covariates will be imputed in cases where these covariates are known risk factors for the outcomes, for example, gender, age or clinical history, and are not missing in more than $50 \%$ of observations in a trial. We will impute median values separately within each trial. Multiple imputation techniques will be used to complete the data required for the primary outcome analysis, and present the data along with the main analysis, as a part of sensitivity analysis. This analysis assumes that missing covariates and endpoints were missing (completely) at random $(\mathrm{M}(\mathrm{C}) \mathrm{AR})$.

\section{Specific analyses performed}

The primary analysis of the primary outcome will be performed using two approaches. (A) A mixed effects logistic regression separately within UC or CD strata. An interaction term of the treatment and subgroup of disease duration is included in the model, following the recommendations on performing meta-analyses of subgroup treatment effects and reducing across-trial confounding. ${ }^{28}$ Specifically, in the one-stage approach: we will use a generalised linear mixed model, accounting for the clustering among patients from the same study by including random study intercept to provide a more conservative estimate of the likelihood of benefit of biologics. ${ }^{34}$ The model includes the treatment-by-duration of disease interaction ${ }^{28}$ and a random effect for the interaction term if duration of disease varies substantially between the studies $(\mathrm{Q}$ $t>1$ ). The link function of the model (logistic, log-binomial, Poisson and negative binomial) is chosen based on the outcome distribution and the model fit. (B) The two-staged approach will also be performed, in which the coefficient for the treatment-by-subgroup interaction within each trial was computed as log odds/relative ratio for the outcome for biologics versus placebo in patients with short disease duration compared with those with long duration of disease. Thereafter, treatment effect measures for the dichotomous outcome of induction of remission in short versus long duration of disease are determined as pooled ratio with $95 \%$ CI of these individual trial coefficient.

As a secondary analysis of the primary outcome, the treatment effect will be adjusted in the model by including the following covariates: exposure to prior anti-TNF, use of concomitant immunomodulators, prior surgery, disease phenotype and extent for $\mathrm{CD}$, disease extent for $\mathrm{UC}$, age, gender, BMI, smoking status, CRP (elevated or not at baseline as per the laboratory normal range in the respective trial), albumin (below normal or not), difference in clinical scores used to measure the efficacy outcomes and the class of the biologic (anti-TNF vs anti-integrins). Covariates are handled by the below mentioned subanalyses, and by meta-regression (if more than 10 studies were available). The procedure PROC NLMIXED will be undertaken to perform the analysis described above. 
Unlike all the above covariates, immunogenicity is not a baseline variable but rather evolves during therapy. Therefore, immunogenicity is not included in the model incorporating baseline patient characteristics.

Secondary analyses include analyses of the response to induction, the maintenance of response, maintenance of remission and the proportion of colectomy (for UC patients only), as well as the subgroup analysis of the primary outcome within the strata of patients treated with anti-TNF class of drugs and patients treated by anti-integrins.

Preplanned sensitivity analyses will assess the primary outcome by the following conditions.

1. Including also trials with high risk of bias.

2. Including only the studies employing the predefined clinical score criterion for remission induction $(\mathrm{CDAI}<150$ or Mayo $\leq 2$ with no individual subscore $>1$ for CD and UC, respectively).

3. Using a fixed-effect model to pool data if heterogeneity assessment reveals $\mathrm{I}^{2}<50 \%$.

4. Including only trials in which all patients were anti-TNF naïve.

5 . Including only patients who rolled over to the maintenance phase after responding to induction (only for the outcome of maintenance of remission, in applicable trials).

6. Analysing the primary outcomes using techniques of Bayesian analysis with vague priors for the treatment effect estimates.

7. Analysing the primary outcomes separately for industry and academic sources.

To assess the optimal threshold for duration of disease that best 'predicts' the likelihood of patients' response to treatment, a summary ROC curve will be constructed based on the random effects generalised linear model adjusting for clustered structure of the pooled database, with the study outcomes as outlined above and continuous disease duration as independent covariate in the model.

SAS V.9.4 and R V.3.2.5 software will be used for the main statistical analysis. We will generate Forest plots of pooled effect estimates (RR or OR) with $95 \%$ CIs, as well as funnel plots. The latter are assessed for evidence of asymmetry and therefore possible publication bias or other small study effects, using the Egger test, if there are sufficient $(n=10)$ eligible studies included in the meta-analysis, in line with published recommendations. ${ }^{33}$

\section{Author affiliations}

${ }^{1}$ Department of Gastroenterology, First affiliated Hospital of the Sun Yatsen University, Guangzhou, China

${ }^{2}$ Department of Gastroenterology, Sheba Medical Center, Sackler School of Medicine, Tel-Aviv University, Tel Aviv, Israel

${ }^{3}$ Department of Public Health, Faculty of Health Sciences, Ben-Gurion University of the Negev, Beer-Sheva, Israel

${ }^{4}$ Department of Gastroenterology, Kitasato Institute Hospital, Tokyo, Japan ${ }^{5}$ Department of Gastroenterology, Rambam Health Care Campus, Bruce \& Ruth Rappaport Faculty of Medicine, Technion Institute of Technology, Haifa, Israel ${ }^{6}$ Helmsley Inflammatory Bowel Disease Center, Icahn School of Medicine, Mount Sinai Hospital, New York City, New York, USA
${ }^{7}$ Department of Gastroenterology, Inserm U594, Nancy University Hospital, Nancy, France

${ }^{8}$ Department of Gastroenterology, University of Calgary, Calgary, Canada

Contributors SB-H conceived and designed the study and drafted the manuscript. $\mathrm{M}$-hC and RM participated in study conception and design and reviewed the manuscript for important intellectual content. YZ and JG collected data and reviewed the manuscript for important intellectual content. LN and RS participated in study design and data analysis and in drafting of the manuscript. JZ, TK, TH, LP-B, JFC, YC and GK participated in study design and reviewed the manuscript for important intellectual content.

Funding This work was supported in part by the 'Talpiot' medical leadership grant from the Sheba Medical Center (to SBH). The funding body provides non-restricted grant, and has no role in developing the protocols nor in participating in project review.

Competing interests SB-H has received consultancy and/or advisory board fees from Abbvie, Novartis, Schering-Plough, Janssen, Celltrion, GSK, Pfizer and Takeda, and research support from Abbvie, Janssen, Celltrion and Takeda. M-hC has received speaker fees from Janssen, Falk, Takeda, AbbVie and Ipson. RM has received speaker fees from Janssen, Falk, Takeda and Ipson. TK received lecture fees from Mitsubishi-Tanabe Pharma, Eisai, Kyorin Pharmaceutical, Abbvie, Janssen, JIMR0, Ajinomoto Pharma, EA Pharma, Astellas, Mochida Pharmaceutical, Asahi Kasei Medical, Takeda Pharmaceutical, Gilead Sciences, Celltrion, Nippon Kayaku, Thermo Fisher Diagnostics; advisory and/or consultancy fees from Janssen, Pfizer, Kyorin Pharmaceutical, Mochida Pharmaceutical, Takeda Pharmaceutical, Eli Lilly, Ferring Pharmaceuticals, Nippon Kayaku, Alfresa Pharma, Research grants EA Pharma, Thermo Fisher Diagnostics, Alfresa Pharma. TH received lecture fees from Mitsubishi-Tanabe Pharma, Kyorin Pharmaceutical, Abbvie GK, Janssen, Mochida Pharmaceutical, JIMRO, Takeda Pharmaceutical, Gilead Sciences, Miyarisan Pharmaceutical, Kissei Pharmaceutical, Zeria Pharmaceutical, Ferring Pharmaceutical, Nippon Kayaku, Advisory/consultancy fees from MitsubishiTanabe Pharma, Takeda Pharmaceutical, EA Pharma, Janssen, Eli Lilly, Pfizer, Zeria Pharmaceutical; Research grant: Abbvie GK, JIMRO, Zeria Pharmaceutical. LP-B received consulting fees from Merck, Abbvie, Janssen, Genentech, Mitsubishi, Ferring, Norgine, Tillots, Vifor, Pharmacosmos, BMS, UCB-pharma, Hospira, Celltrion, Takeda, Biogaran, Boehringer-Ingelheim, Lilly, Pfizer, HAC-Pharma, Index Pharmaceuticals, Amgen, Sandoz, Forward Pharma GmbH, Celgene, Biogen, Lycera, Samsung Bioepis. Lecture fees from Merck, Abbvie, Takeda, Janssen, Ferring, Norgine, Tillots, Vifor, Mitsubishi, HAC-pharma. YC received research grants, speaker and advisory fees from Abbvie, speaker and advisor fees from Janssen, research grant, advisory fees from Medtronics, and Protalix, speaker and advisory fees from Takeda. GK has served as a speaker for Janssen, Merck, ScheringPlough, Abbvie and UCB Pharma, on advisory board for Janssen, Abbvie, Merck, Schering-Plough, Shire and UCB Pharma and has received research support from Merck, Abbvie, GlaxoSmithKline and Shire. JFC is a consultant, advisory board member and speaker for AbbVie, BMS, Ferring, Genentech, Giuliani, Given Imaging, Merck, Millennium, Pfizer, Prometheus, Sanofi, Schering Plough, Takeda, Teva and UCB Pharma.

\section{Patient consent for publication Not required.}

Ethics approval This meta-analysis was approved by the Ethics Committee of the First Affiliated Hospital of Sun Yat-sen University. Findings will be published in peerreviewed journal and disseminated via scientific meetings and links with patients organisations .

Provenance and peer review Not commissioned; externally peer reviewed.

Open access This is an open access article distributed in accordance with the Creative Commons Attribution Non Commercial (CC BY-NC 4.0) license, which permits others to distribute, remix, adapt, build upon this work non-commercially, and license their derivative works on different terms, provided the original work is properly cited, appropriate credit is given, any changes made indicated, and the use is non-commercial. See: http://creativecommons.org/licenses/by-nc/4.0/.

\section{REFERENCES}

1. Peyrin-Biroulet L, Billioud V, D'Haens G, et al. Development of the Paris definition of early Crohn's disease for disease-modification trials: results of an international expert opinion process. Am J Gastroenterol 2012;107:1770-6.

2. D'Haens GR, Panaccione R, Higgins PD, et al. The London Position Statement of the World Congress of Gastroenterology on Biological 
Therapy for IBD with the European Crohn's and Colitis Organization: when to start, when to stop, which drug to choose, and how to predict response? Am J Gastroenterol 2011;106:199-212.

3. D'Haens G, Baert F, van Assche G, et al. Early combined immunosuppression or conventional management in patients with newly diagnosed Crohn's disease: an open randomised trial. Lancet 2008;371:660-7.

4. Khanna R, Bressler B, Levesque BG, et al. Early combined immunosuppression for the management of Crohn's disease (REACT): a cluster randomised controlled trial. Lancet 2015;386:1825-34

5. Schreiber S, Colombel JF, Bloomfield R, et al. Increased response and remission rates in short-duration Crohn's disease with subcutaneous certolizumab pegol: an analysis of PRECiSE 2 randomized maintenance trial data. Am J Gastroenterol 2010;105:1574-82.

6. Schreiber S, Reinisch W, Colombel JF, et al. Subgroup analysis of the placebo-controlled CHARM trial: Increased remission rates through 3years for adalimumab-treated patients with early Crohn's disease. $J$ Crohns Colitis 2013;7:213-21.

7. Ma C, Beilman CL, Huang VW, et al. Anti-TNF Therapy Within 2 Years of Crohn's Disease Diagnosis Improves Patient Outcomes: A Retrospective Cohort Study. Inflamm Bowel Dis 2016;22:870-9.

8. Kotze PG, Ludvig JC, Teixeira FV, et al. Disease duration did not influence the rates of loss of efficacy of the anti-TNF therapy in Latin American Crohn's disease patients. Digestion 2015;91:158-63.

9. Cochrane Handbook for Systematic Reviews of Interventions. Version 5.1.0. http://handbook.cochrane.org/ (Accessed 17 Oct 2015).

10. Moher D, Liberati A, Tetzlaff J, et al. Preferred reporting items for systematic reviews and meta-analyses: the PRISMA Statement. $J$ Clin Epidemiol 2009;62:1006-12.

11. Moher D, Shamseer L, Clarke M, et al. Preferred reporting items for systematic review and meta-analysis protocols (PRISMA-P) 2015 statement. Syst Rev 2015;4:1.

12. Colombel JF, Sandborn WJ, Reinisch W, et al. Infliximab, azathioprine, or combination therapy for Crohn's Disease. N Engl J Med Overseas Ed 2010;362:1383-95.

13. Ananthakrishnan AN, Binion DG. Editorial: Improved Efficacy of Biological Maintenance Therapy in "Early" Compared With "Late" Crohn's Disease: Strike While the Iron Is Hot With Anti-TNF Agents? Am J Gastroenterol 2010;105:1583-5.

14. Nuij V, Fuhler GM, Edel AJ, et al. Benefit of Earlier Anti-TNF Treatment on IBD Disease Complications? J Crohns Colitis 2015;9:997-1003.

15. Safroneeva E, Vavricka SR, Fournier N, et al. Impact of the early use of immunomodulators or TNF antagonists on bowel damage and surgery in Crohn's disease. Aliment Pharmacol Ther 2015;42:977-89.

16. Oussalah A, Evesque L, Laharie D, et al. A multicenter experience with infliximab for ulcerative colitis: outcomes and predictors of response, optimization, colectomy, and hospitalization. Am J Gastroenterol 2010;105:2617-25.

17. Matsumoto T, lida M, Motoya S, et al. Therapeutic efficacy of infliximab on patients with short duration of Crohn's disease: a Japanese multicenter survey. Dis Colon Rectum 2008;51:916-23.
18. Peyrin-Biroulet L, Billioud V, D'Haens G, et al. Development of the paris definition of early crohn's disease for disease-modification trials: results of an international expert opinion process. Am J Gastroenterol 2012;107:1770-6.

19. Bickston SJ, Behm BW, Tsoulis DJ, et al. Vedolizumab for induction and maintenance of remission in ulcerative colitis. Cochrane Database Syst Rev 2014:CD007571.

20. MacDonald JK, McDonald JW. Natalizumab for induction of remission in Crohn's disease. Cochrane Database Syst Rev 2007:CD006097.

21. Behm BW, Bickston SJ. Tumor necrosis factor-alpha antibody for maintenance of remission in Crohn's disease. Cochrane Database Syst Rev 2008:CD006893.

22. Lawson MM, Thomas AG, Akobeng AK. Tumour necrosis factor alpha blocking agents for induction of remission in ulcerative colitis. Cochrane Database Syst Rev 2006:CD005112.

23. Dassopoulos T, Sultan S, Falck-Ytter YT, et al. American Gastroenterological Association Institute Technical Review on the Use of Thiopurines, Methotrexate, and Anti-TNF- $\alpha$ Biologic Drugs for the Induction and Maintenance of Remission in Inflammatory Crohn's Disease. Gastroenterology 2013;145:1464-78.

24. Dignass A, Lindsay JO, Sturm A, et al. Second European evidence-based consensus on the diagnosis and management of ulcerative colitis Part 2: Current management. J Crohns Colitis 2012;6:991-1030.

25. Dignass A, Van Assche G, Lindsay JO, et al. The second European evidence-based Consensus on the diagnosis and management of Crohn's disease: Current management. J Crohns Colitis 2010;4:28-62.

26. Higgins JPT. Cochrane handbook for systematic reviews of interventions: version 5.0.0, 2008.

27. Guyatt $\mathrm{GH}$, Oxman AD, Montori V, et al. GRADE guidelines: 5 . Rating the quality of evidence-publication bias. J Clin Epidemiol 2011;64:1277-82.

28. Thompson S, Higgins J. Can meta-analysis help target interventions at individuals most likely to benefit? The Lancet 2005;365:341-6.

29. Tierney JF, Vale C, Riley R, et al. IPD) Meta-analyses of Randomized Controlled Trials: Guidance on Their Use. PLoS Med 2015;12:e1001855

30. Riley RD, Lambert PC, Staessen JA, et al. Meta-analysis of continuous outcomes combining individual patient data and aggregate data. Stat Med 2008;27:1870-93.

31. Debray TPA, Moons KGM, van Valkenhoef $G$, et al. Get real in individual participant data (IPD) meta-analysis: a review of the methodology. Res Synth Methods 2015;6:293-309.

32. Simmonds MC, Higgins JPT. Covariate heterogeneity in metaanalysis: Criteria for deciding between meta-regression and individual patient data. Stat Med 2007;26:2982-99.

33. Higgins JPT, Thompson SG, Deeks JJ, Altman DG. Measuring inconsistency in meta-analyses. BMJ 2003;327:557-60.

34. DerSimonian R, Laird N. Meta-analysis in clinical trials. Control Clin Trials 1986;7:177-88. 